PROCEEDINGS OF THE

AMERICAN MATHEMATICAL SOCIETY

Volume 131, Number 7, Pages 2037-2042

S 0002-9939(03)06765-0

Article electronically published on January 28, 2003

\title{
A DIRECT PROOF OF THE QUANTUM VERSION OF MONK'S FORMULA
}

\author{
ANDERS SKOVSTED BUCH
}

(Communicated by John R. Stembridge)

\begin{abstract}
We use classical Schubert calculus to give a direct geometric proof of the quantum version of Monk's formula.
\end{abstract}

\section{INTRODUCTION}

The quantum version of Monk's formula of Fomin, Gelfand, and Postnikov [6] gives an explicit rule for multiplying by a codimension one Schubert class in the (small) quantum cohomology ring of a flag variety $S L_{n} / B$. The proof given in 6] relies on a formula of Ciocan-Fontanine 3] for the quantum classes of certain special Schubert varieties given by cyclic permutations, which is obtained using degeneracy loci formulas on hyper-quot schemes. In the present paper we give a direct geometric proof of the quantum Monk's formula which relies only on classical Schubert calculus and the definition of Gromov-Witten invariants. In particular, no compactifications of moduli spaces are required. Our proof uses an adaption of the ideas from [1] where we give a similar proof of the quantum Pieri formula for Grassmann varieties.

Since the quantum cohomology ring of a flag variety is generated by the codimension one Schubert classes, the quantum Monk's formula uniquely determines this ring as well as the associated Gromov-Witten invariants. Thus, if associativity of quantum cohomology is granted [16, 12, 9, we obtain a completely elementary understanding of this ring.

The presentation of the quantum cohomology ring of a flag variety due to Givental, Kim, and Ciocan-Fontanine [10, 11, 3] and Ciocan-Fontanine's formula for special quantum Schubert classes 3 are easy consequences of the quantum Monk's formula. In fact, the quantum Monk's formula implies that Ciocan-Fontanine's classes satisfy the same recursive relations as those defining the quantum elementary symmetric polynomials (cf. 15, Lemma 4.2]). These results in turn are the only facts required in the combinatorial proof of the quantum Giambelli formula for flag varieties given in [6]. Alternatively, the quantum Schubert polynomials constructed in [6] can easily be computed by using only the quantum Monk's formula (cf. [6, §8] and [13, (4.16)]). The quantum Pieri formula of Ciocan-Fontanine [4]

Received by the editors December 18, 2001 and, in revised form, February 22, 2002 and March $6,2002$.

2000 Mathematics Subject Classification. Primary 14N35; Secondary 14M15.

The author was partially supported by NSF Grant DMS-0070479.

(C)2003 American Mathematical Society 
can also be derived combinatorially from the quantum Monk's formula 15, 7], 1] or it can be proved by an enhancement of the methods of the present paper [2]. For a survey of combinatorial approaches to quantum cohomology of flag varieties we refer the reader to [5].

In Section 2 we fix notation regarding Schubert varieties in partial flag varieties and prove a result which relates the Schubert varieties in different partial flag varieties. In Section 3 we give some tools for handling rational curves in flag varieties. The proof of the quantum Monk's formula is finally given in Section 4 after a short introduction of the quantum ring of a flag variety.

We thank S. Fomin for helpful comments regarding our paper.

\section{SCHUBERT VARIETIES IN PARTIAL FLAG VARIETIES}

Our notation for Schubert varieties is based on $\left[8\right.$. Set $E=\mathbb{C}^{n}$ and let $\mathrm{F} \ell(E)=$ $\left\{V_{1} \subset V_{2} \subset \cdots \subset V_{n-1} \subset E \mid \operatorname{dim} V_{i}=i\right\}$ denote the variety of full flags in $E$. Given a fixed flag $F_{1} \subset F_{2} \subset \cdots \subset F_{n-1} \subset E$ and a permutation $w \in S_{n}$ there is a Schubert variety

$$
\Omega_{w}\left(F_{\bullet}\right)=\left\{V_{\bullet} \in \mathrm{F} \ell(E) \mid \operatorname{dim}\left(V_{p} \cap F_{q}\right) \geq p-r_{w}(p, n-q) \forall p, q\right\}
$$

where $r_{w}(p, q)=\#\{i \leq p \mid w(i) \leq q\}$. The codimension of this variety is equal to the length $\ell(w)$ of $w$. Notice that the rank conditions on $V_{p}$ are redundant unless $w$ has a descent at position $p$, i.e. $w(p)>w(p+1)$.

Given a sequence of integers $a=\left(a_{1} \leq a_{2} \leq \cdots \leq a_{k}\right)$ with $a_{1} \geq 0$ and $a_{k} \leq n$, we have the partial flag variety $\mathrm{F} \ell(a ; E)=\left\{V_{a_{1}} \subset \cdots \subset V_{a_{k}} \subset E \mid \operatorname{dim} V_{a_{i}}=a_{i}\right\}$. Although all such varieties can be obtained from strictly increasing sequences $a$, it will be convenient to allow weakly increasing sequences in the notation. Similarly it is useful to set $a_{0}=0$ and $a_{k+1}=n$. Let $S_{n}(a) \subset S_{n}$ denote the set of permutations whose descent positions are contained in the set $\left\{a_{1}, a_{2}, \ldots, a_{k}\right\}$. The Schubert varieties in $\mathrm{F} \ell(a ; E)$ are indexed by these permutations; the Schubert variety corresponding to $w \in S_{n}(a)$ is given by

$$
\Omega_{w}^{(a)}\left(F_{\bullet}\right)=\left\{V_{\bullet} \in \mathrm{F} \ell(a ; E) \mid \operatorname{dim}\left(V_{a_{i}} \cap F_{q}\right) \geq a_{i}-r_{w}\left(a_{i}, n-q\right) \forall i, q\right\} .
$$

Let $\rho_{a}: \mathrm{F} \ell(E) \rightarrow \mathrm{F} \ell(a ; E)$ be the projection which maps a full flag $V_{\bullet}$ to the subflag $V_{a_{1}} \subset \cdots \subset V_{a_{k}}$. Then for any $w \in S_{n}(a)$ we have $\rho_{a}^{-1}\left(\Omega_{w}^{(a)}\left(F_{\bullet}\right)\right)=\Omega_{w}\left(F_{\bullet}\right)$. On the other hand, if $w \in S_{n}$ is any permutation, then $\rho_{a}\left(\Omega_{w}\left(F_{\bullet}\right)\right)=\Omega_{\widetilde{w}}^{(a)}\left(F_{\bullet}\right)$ where $\widetilde{w} \in S_{n}(a)$ is the permutation obtained from $w$ by rearranging the elements $w\left(a_{i}+1\right), w\left(a_{i}+2\right), \ldots, w\left(a_{i+1}\right)$ in increasing order for each $0 \leq i \leq k$. In other words, $\widetilde{w}$ is the shortest representative for $w$ modulo the subgroup $W_{a} \subset S_{n}$ generated by the simple reflections $s_{i}=(i, i+1)$ for $i \notin\left\{a_{1}, \ldots, a_{k}\right\}$. For example, if $n=6, a=(2,5)$, and $w=623154$, then $\widetilde{w}=261354$.

Now let $b=\left(b_{1} \leq b_{2} \leq \cdots \leq b_{k}\right)$ be another sequence with the same length as $a$, such that $b_{i} \leq a_{i}$ for each $i$. Given a permutation $w \in S_{n}(a)$ we will need a description of the set $\left\{K_{\bullet} \in \mathrm{F} \ell(b ; E) \mid \exists V_{\bullet} \in \Omega_{w}^{(a)}\left(F_{\bullet}\right): K_{b_{i}} \subset V_{a_{i}} \forall i\right\}$.

We construct a permutation $\bar{w} \in S_{n}(b)$ from $w$ as follows. Set $w^{(0)}=w$. Then for each $1 \leq i \leq k$ we let $w^{(i)}$ be the permutation obtained from $w^{(i-1)}$ by rearranging the elements $w^{(i-1)}\left(b_{i}+1\right), \ldots, w^{(i-1)}\left(a_{i+1}\right)$ in increasing order. Finally we set $\bar{w}=w^{(k)}$. For example, if $n=6, a=(2,5), b=(1,2)$, and $w=263451$, then $w^{(1)}=234561$ and $\bar{w}=231456$.

\footnotetext{
${ }^{1}$ Ciocan-Fontanine's result is more general and covers all partial flag varieties $S L_{n} / P$.
} 
Lemma 1. The set $\left\{K_{\bullet} \in \mathrm{F} \ell(b ; E) \mid \exists V_{\bullet} \in \Omega_{w}^{(a)}\left(F_{\bullet}\right): K_{b_{i}} \subset V_{a_{i}} \forall i\right\}$ is equal to the Schubert variety $\Omega_{w}^{(b)}\left(F_{\bullet}\right)$ in $\mathrm{F} \ell(b ; E)$.

Proof. We prove that the subset $\Omega_{i}$ of $\mathrm{F} \ell_{i}=\mathrm{F} \ell\left(b_{1}, \ldots, b_{i}, a_{i+1}, \ldots, a_{k} ; E\right)$ defined by $\Omega_{i}=\left\{K_{\bullet} \mid \exists V_{\bullet} \in \Omega_{w}^{(a)}\left(F_{\bullet}\right): K_{b_{j}} \subset V_{a_{j}}\right.$ for $j \leq i$ and $K_{a_{j}}=V_{a_{j}}$ for $\left.j>i\right\}$ is equal to the Schubert variety in $\mathrm{F} \ell_{i}$ given by the permutation $w^{(i)}$. This is true when $i=0$. Let $\rho_{j}: \mathrm{F} \ell(E) \rightarrow \mathrm{F} \ell_{j}$ denote the projection. Then it is easy to check that $\Omega_{i+1}=\rho_{i+1}\left(\rho_{i}^{-1}\left(\Omega_{i}\right)\right)$, so the lemma follows from the above remarks about images and inverse images of projections $\rho_{a}$.

Lemma 1 has a dual version which we will also need. Let $a$ and $c$ be weakly increasing sequences of integers between 0 and $n$, each of length $k$, such that $a_{i} \leq c_{i}$ for each $1 \leq i \leq k$. Given $w \in S_{n}(a)$ we define a permutation $\widehat{w} \in S_{n}(c)$ as follows. Set $w^{(k+1)}=w$. For each $i=k, k-1, \ldots, 1$ we then let $w^{(i)}$ be the permutation obtained from $w^{(i+1)}$ by rearranging the elements $w^{(i+1)}\left(a_{i-1}+1\right), \ldots, w^{(i+1)}\left(c_{i}\right)$ in increasing order. Finally we set $\widehat{w}=w^{(1)}$.

Lemma 2. The set $\left\{W_{\bullet} \in \mathrm{F} \ell(c ; E) \mid \exists V_{\bullet} \in \Omega_{w}^{(a)}\left(F_{\bullet}\right): V_{a_{i}} \subset W_{c_{i}} \forall i\right\}$ is equal to the Schubert variety $\Omega_{\widehat{w}}^{(c)}\left(F_{\bullet}\right)$ in $\mathrm{F} \ell(c ; E)$.

Notice that the definitions of the permutations $\bar{w}$ and $\widehat{w}$ imply that $\ell(\bar{w}) \geq \ell(w)-$ $\sum_{i=1}^{k}\left(a_{i}-b_{i}\right)\left(a_{i+1}-a_{i}\right)$ and $\ell(\widehat{w}) \geq \ell(w)-\sum_{i=1}^{k}\left(c_{i}-a_{i}\right)\left(a_{i}-a_{i-1}\right)$. In particular, if $a=(1,2, \ldots, n-1)$ so that $\mathrm{F} \ell(a ; E)=\mathrm{F} \ell(E)$, then $\ell(\bar{w}) \geq \ell(w)-\sum_{i=1}^{n-1}\left(i-b_{i}\right)$ and $\ell(\widehat{w}) \geq \ell(w)-\sum_{i=1}^{n-1}\left(c_{i}-i\right)$.

\section{RATiOnAL CURVES IN PARTIAL FLAG VARIETIES}

By a rational curve in $\mathrm{F} \ell(a ; E)$ we will mean the image $C$ of a regular function $\mathbb{P}^{1} \rightarrow \mathrm{F} \ell(a ; E)$. (We will tolerate that a rational curve can be a point according to this definition.) Given a rational curve $C \subset \mathrm{F} \ell(a ; E)$ we let $C_{i}=\rho_{a_{i}}(C) \subset \operatorname{Gr}\left(a_{i}, E\right)$ be the image of $C$ by the projection $\rho_{a_{i}}: \mathrm{F} \ell(a ; E) \rightarrow \operatorname{Gr}\left(a_{i}, E\right)$. This curve $C_{i}$ then has a kernel and a span 1 . The kernel is the largest subspace of $E$ contained in all the $a_{i}$-dimensional subspaces of $E$ corresponding to points of $C_{i}$. We let $b_{i}$ be the dimension of this kernel and denote the kernel itself by $K_{b_{i}}$. Similarly, the span of $C_{i}$ is the smallest subspace of $E$ containing all the subspaces given by points of $C_{i}$. We let $c_{i}$ be the dimension of this span and denote the span by $W_{c_{i}}$. These subspaces define partial flags $K_{\bullet} \in \mathrm{F} \ell(b ; E)$ and $W_{\bullet} \in \mathrm{F} \ell(c ; E)$ where $b=\left(b_{1} \ldots, b_{k}\right)$ and $c=\left(c_{1}, \ldots, c_{k}\right)$, which we will call the kernel and span of $C$.

Proposition 1. Let $C \subset \mathrm{F} \ell(a ; E)$ be a rational curve with kernel $K_{\bullet} \in \mathrm{F} \ell(b ; E)$ and span $W_{\bullet} \in F l(c ; E)$ and let $w \in S_{n}(a)$. If $C \cap \Omega_{w}^{(a)}\left(F_{\bullet}\right) \neq \emptyset$, then $K_{\bullet} \in \Omega_{\bar{w}}^{(b)}\left(F_{\bullet}\right)$ and $W_{\bullet} \in \Omega_{\widehat{w}}^{(c)}\left(F_{\bullet}\right)$.

Proof. If $V_{\bullet} \in C \cap \Omega_{w}^{(a)}\left(F_{\bullet}\right)$, then we have $K_{b_{i}} \subset V_{a_{i}} \subset W_{c_{i}}$ for all $i$. The proposition therefore follows from Lemma 1 and Lemma 2

Now let $a=\left(a_{1}<a_{2}<\cdots<a_{k}\right)$ be a strictly increasing sequence of integers with $1 \leq a_{i} \leq n-1$. Define the multidegree of a rational curve $C \subset \mathrm{F} \ell(a ; E)$ to be the sequence $d=\left(d_{1}, \ldots, d_{k}\right)$ where $d_{i}$ is the number of points in the intersection $C \cap \Omega_{s_{a_{i}}}\left(F_{\bullet}\right)$ for any flag $F_{\text {• }}$ in general position. Notice that $d_{i}$ is greater than or equal to the degree of the image $C_{i} \subset \operatorname{Gr}\left(a_{i} ; E\right)$. If $K \bullet \in \mathrm{F} \ell(b ; E)$ is the kernel and 
$W_{\bullet} \in \mathrm{F} \ell(c ; E)$ the span of $C$, it therefore follows from [1, Lemma 1] that $b_{i} \geq a_{i}-d_{i}$ and $c_{i} \leq a_{i}+d_{i}$ for all $1 \leq i \leq k$.

Next we shall need a fact about rational curves in the full flag variety $\mathrm{F} \ell(E)$. For integers $1 \leq i<j \leq n$, let $d_{i j}=(0, \ldots, 0,1, \ldots, 1,0, \ldots, 0)$ denote the multidegree consisting of $i-1$ zeros followed by $j-i$ ones followed by $n-j$ zeros, i.e. $\left(d_{i j}\right)_{p}=1$ for $i \leq p<j$ and $\left(d_{i j}\right)_{p}=0$ otherwise. We set $a=(1,2, \ldots, n-1)$ and $b=$ $a-d_{i j}=\left(b_{1}, \ldots, b_{n-1}\right)$ where $b_{p}=p-\left(d_{i j}\right)_{p}$.

Proposition 2. Let $K_{\bullet} \in \mathrm{F} \ell(b ; E)$ and let $W \subset E$ be a subspace of dimension $i+1$ such that $K_{j-2} \cap W=K_{i-1}$ and $K_{j-2}+W=K_{j}$. Then there exists a unique rational curve $C \subset \mathrm{F} \ell(E)$ of multidegree $d_{i j}$ such that $K$. is the kernel of $C$ and $W$ is the span of $C_{i} \subset \operatorname{Gr}(i, E)$.

Proof. The only curve satisfying the conditions of the proposition is the set of flags

$$
V_{\bullet}=\left(K_{1} \subset \cdots \subset K_{i-1} \subset L \subset K_{i}+L \subset \cdots \subset K_{j-2}+L \subset K_{j} \subset \cdots \subset K_{n-1}\right)
$$

for all $i$-dimensional subspaces $L$ such that $K_{i-1} \subset L \subset W$.

It is easy to show that the rational curves $C \subset \mathrm{F} \ell(E)$ of multidegree $d_{i j}$ are in fact in 1-1 correspondence with the pairs $\left(K_{\bullet}, W\right)$ of the proposition, but we shall not need this fact.

\section{Quantum COHOMOlOGY OF FLAG VARIETIES}

For each permutation $w \in S_{n}$ we let $\Omega_{w}$ denote the class of $\Omega_{w}\left(F_{\bullet}\right)$ in the cohomology ring $H^{*} \mathrm{~F} \ell(E)=H^{*}(\mathrm{~F} \ell(E) ; \mathbb{Z})$. The Schubert classes $\Omega_{w}$ form a basis for this ring. If $d=\left(d_{1}, \ldots, d_{n-1}\right)$ is a multidegree we set $|d|=\sum d_{i}$. Given three permutations $u, v, w \in S_{n}$ such that $\ell(u)+\ell(v)+\ell(w)=\left(\begin{array}{c}n \\ 2\end{array}\right)+2|d|$, the Gromov-Witten invariant $\left\langle\Omega_{u}, \Omega_{v}, \Omega_{w}\right\rangle_{d}$ is defined as the number of rational curves of multidegree $d$ in $\mathrm{F} \ell(E)$ which meet each of the Schubert varieties $\Omega_{u}\left(F_{\bullet}\right), \Omega_{v}\left(G_{\bullet}\right)$, and $\Omega_{w}\left(H_{\bullet}\right)$ for general fixed flags $F_{\bullet}, G_{\bullet}, H_{\bullet}$ in $E$. If $\ell(u)+\ell(v)+\ell(w) \neq\left(\begin{array}{l}n \\ 2\end{array}\right)+2|d|$, then $\left\langle\Omega_{u}, \Omega_{v}, \Omega_{w}\right\rangle_{d}=0$.

Let $q_{1}, \ldots, q_{n-1}$ be independent variables, and write $\mathbb{Z}[q]=\mathbb{Z}\left[q_{1}, \ldots, q_{n-1}\right]$. The quantum cohomology ring $Q H^{*} \mathrm{~F} \ell(E)$ is a $\mathbb{Z}[q]$-algebra which is isomorphic to $H^{*} \mathrm{~F} \ell(E) \otimes \mathbb{Z}[q]$ as a module over $\mathbb{Z}[q]$. In this ring we have quantum Schubert classes $\sigma_{w}=\Omega_{w} \otimes 1$. Multiplication in $Q H^{*} \mathrm{~F} \ell(E)$ is defined by

$$
\sigma_{u} \cdot \sigma_{v}=\sum_{w, d}\left\langle\Omega_{u}, \Omega_{v}, \Omega_{w^{\vee}}\right\rangle_{d} q^{d} \sigma_{w}
$$

where the sum is over all permutations $w \in S_{n}$ and multidegrees $d=\left(d_{1}, \ldots, d_{n-1}\right)$; here we set $q^{d}=\prod q_{i}^{d_{i}}$ and we let $w^{\vee} \in S_{n}$ denote the permutation of the dual Schubert class to $\Omega_{w}$, i.e. $w^{\vee}=w_{0} w$ where $w_{0}$ is the longest permutation in $S_{n}$. It is a non-trivial fact that this defines an associative ring [16, 12, 9].

For $1 \leq i<j \leq n$ we let $t_{i j}=(i, j) \in S_{n}$ denote the transposition which interchanges $i$ and $j$. We furthermore set $q_{i j}=q^{d_{i j}}=q_{i} q_{i+1} \ldots q_{j-1}$. Our goal is to prove the following quantum version of the Monk's formula from [6].

Theorem 1. For $w \in S_{n}$ and $1 \leq r<n$ we have

$$
\sigma_{s_{r}} \cdot \sigma_{w}=\sum \sigma_{w t_{k l}}+\sum q_{i j} \sigma_{w t_{i j}}
$$


where the first sum is over all transpositions $t_{k l}$ such that $k \leq r<l$ and $\ell\left(w t_{k l}\right)=$ $\ell(w)+1$, and the second sum is over all transpositions $t_{i j}$ such that $i \leq r<j$ and $\ell\left(w t_{i j}\right)=\ell(w)-\ell\left(t_{i j}\right)=\ell(w)-2(j-i)+1$.

Proof. The first sum is dictated by the classical Monk's formula [14. The second sum is equivalent to the following statement. If $d=\left(d_{1}, \ldots, d_{n-1}\right)$ is a non-zero multidegree and $u, w \in S_{n}$ are permutations such that $\ell(u)+\ell(w)+\ell\left(s_{r}\right)=\left(\begin{array}{l}n \\ 2\end{array}\right)+$ $2|d|$, then the Gromov-Witten invariant $\left\langle\Omega_{u}, \Omega_{w}, \Omega_{s_{r}}\right\rangle_{d}$ is equal to one if $d=d_{i j}$ for some $i, j$ such that $i \leq r<j$ and $u^{-1} w_{0} w=t_{i j}$; otherwise $\left\langle\Omega_{u}, \Omega_{w}, \Omega_{s_{r}}\right\rangle_{d}=0$.

Suppose $\left\langle\Omega_{u}, \Omega_{w}, \Omega_{s_{r}}\right\rangle_{d} \neq 0$ and let $C$ be a rational curve of multidegree $d$ which meets three Schubert varieties $\Omega_{u}\left(F_{\bullet}\right), \Omega_{w}\left(G_{\bullet}\right)$, and $\Omega_{s_{r}}\left(H_{\bullet}\right)$ in general position. Let $K_{\bullet} \in \mathrm{F} \ell(b ; E)$ be the kernel of $C$ and set $a=(1,2, \ldots, n-1)$. Then $b_{p} \geq a_{p}-d_{p}$ for all $1 \leq p \leq n-1$. By Proposition 1 we have $K_{\bullet} \in \Omega_{\bar{u}}^{(b)}\left(F_{\bullet}\right) \cap \Omega \frac{(b)}{w}\left(G_{\bullet}\right) \cap \Omega_{\frac{(b)}{s_{r}}}\left(H_{\bullet}\right)$. Since the flags are general this implies that $\ell(\bar{u})+\ell(\bar{w})+\ell\left(\overline{s_{r}}\right) \leq \operatorname{dim} \mathrm{F} \ell(b ; E)$. On the other hand the inequalities $\ell(\bar{u}) \geq \ell(u)-\sum\left(p-b_{p}\right), \ell(\bar{w}) \geq \ell(w)-\sum\left(p-b_{p}\right)$, $\ell\left(\overline{s_{r}}\right) \geq 0$, and $\sum\left(p-b_{p}\right) \leq|d|$ imply that $\ell(\bar{u})+\ell(\bar{w})+\ell\left(\overline{s_{r}}\right) \geq\left(\begin{array}{l}n \\ 2\end{array}\right)-1$. Since this is the maximal possible dimension of $\mathrm{F} \ell(b ; E)$ we conclude that all inequalities are satisfied with equality.

This first implies that $b=a-d=\left(1-d_{1}, 2-d_{2}, \ldots, n-1-d_{n-1}\right)$. Furthermore, since $\operatorname{dim} \mathrm{F} \ell(b ; E)=\left(\begin{array}{l}n \\ 2\end{array}\right)-1$ we deduce that $d=d_{i j}$ for some $1 \leq i<j \leq n$. Thus $\mathrm{F} \ell(b ; E)=\mathrm{F} \ell(1, \ldots, j-2, j, \ldots, n-1 ; E)$ is the variety of partial flags with subspaces of all dimensions other than $j-1$. Since $\ell\left(\overline{s_{r}}\right)=0$ it follows that $i \leq r<j$. The fact that $\ell(\bar{u})=\ell(u)-|d|$ implies that $\bar{u}=u s_{i} s_{i+1} \cdots s_{j-1}$ by the definition of $\bar{u}$. Similarly we have $\bar{w}=w s_{i} s_{i+1} \cdots s_{j-1}$. Now since $\ell(\bar{u})+\ell(\bar{w})=\operatorname{dim} \mathrm{F} \ell(b ; E)$ and $\Omega_{\bar{u}}^{(b)}\left(F_{\bullet}\right) \cap \Omega_{\bar{w}}^{(b)}\left(G_{\bullet}\right) \neq \emptyset$ we conclude that $\bar{u}$ and $\bar{w}$ are dual with respect to $\mathrm{F} \ell(b ; E)$, i.e. $\bar{u}^{-1} w_{0} \bar{w}=s_{j-1}$ or equivalently $u^{-1} w_{0} w=t_{i j}$ as required.

It remains to be proved that if $d=d_{i j}$ and $u^{-1} w_{0} w=t_{i j}$ for some $i \leq r<j$, then there exists a unique rational curve of multidegree $d$ which meets the three given Schubert varieties. Set $\bar{u}=u s_{i} s_{i+1} \cdots s_{j-1}$ and $\bar{w}=w s_{i} s_{i+1} \cdots s_{j-1}$. Since $\ell\left(u t_{i j}\right)=\ell\left(w_{0} w\right)=\left(\begin{array}{c}n \\ 2\end{array}\right)-\ell(w)=\ell(u)-\ell\left(t_{i j}\right)$ it follows that $\ell(\bar{u})=\ell(u)-|d|$ and similarly $\ell(\bar{w})=\ell(w)-|d|$. Thus $\ell(\bar{u})+\ell(\bar{w})=\operatorname{dim} \mathrm{F} \ell(b ; E)$ where $b=a-d$. Since $\bar{u}^{-1} w_{0} \bar{w}=s_{j-1}$ we conclude that there is a unique partial flag $K_{\bullet} \in \Omega_{\bar{u}}^{(b)}\left(F_{\bullet}\right) \cap$ $\Omega_{\bar{w}}^{(b)}\left(G_{\bullet}\right)$. Similarly, if we set $\widehat{u}=u s_{j-1} s_{j-2} \cdots s_{i}$ and $\widehat{w}=w s_{j-1} s_{j-2} \cdots s_{i}$, then there exists a unique partial flag $W_{\bullet} \in \Omega_{\widehat{u}}^{(c)}\left(F_{\bullet}\right) \cap \Omega_{\widehat{w}}^{(c)}\left(G_{\bullet}\right)$ where $c=a+d$.

In fact, we can say precisely what these partial flags look like. For $1 \leq p \leq n$ we set $L_{p}=F_{n+1-p} \cap G_{p}$. Since the flags $F_{\bullet}$ and $G_{\bullet}$ are general, these spaces have dimension one, and $E=L_{1} \oplus \cdots \oplus L_{n}$. Now $K_{p}=L_{\bar{u}(1)} \oplus \cdots \oplus L_{\bar{u}(p)}$ for each $p \neq j-1$ and $W_{p}=L_{\widehat{u}(1)} \oplus \cdots \oplus L_{\widehat{u}(p)}$ for $p \neq i$. Otherwise stated we have $K_{p}=W_{p}=L_{u(1)} \oplus \cdots \oplus L_{u(p)}$ for $1 \leq p \leq i-1$ and for $j \leq p<n$. For $i-1 \leq p \leq$ $j-2$ we have $K_{p}=K_{i-1} \oplus L_{u(i+1)} \oplus \cdots \oplus L_{u(p+1)}$ while $W_{p+2}=K_{p} \oplus U$ where $U=L_{u(i)} \oplus L_{u(j)}$. In particular we get $W_{i+1} \cap K_{j-2}=K_{i-1}$ and $W_{i+1}+K_{j-2}=K_{j}$ so by Proposition 2 there is exactly one rational curve of multidegree $d$ with kernel $K_{\text {. }}$ and span $W_{\text {. }}$. This curve consists of all flags

$$
V_{\bullet}=\left(K_{1} \subset \cdots \subset K_{i-1} \subset K_{i-1} \oplus L \subset \cdots \subset K_{j-2} \oplus L \subset K_{j} \subset \cdots \subset K_{n-1}\right)
$$

where $L \subset U$ is a one-dimensional subspace. When $L=L_{u(i)}$ we have $V_{\bullet} \in \Omega_{u}\left(F_{\bullet}\right)$, while $V_{\bullet} \in \Omega_{w}\left(G_{\bullet}\right)$ when $L=L_{u(j)}$. Finally, $V_{\bullet}$ belongs to $\Omega_{s_{r}}\left(H_{\bullet}\right)$ if and only if $V_{r} \cap H_{n-r} \neq 0$. Now take any non-zero element $x \in W_{r+1} \cap H_{n-r}$ and let $x^{\prime}$ be 
the $U$-component of $x$ in $W_{r+1}=K_{r-1} \oplus U$. Taking $L=\mathbb{C} x^{\prime}$ then gives a point $V_{\bullet} \in \Omega_{s_{r}}\left(H_{\bullet}\right)$. This completes the proof.

\section{REFERENCES}

[1] A. S. Buch, Quantum cohomology of Grassmannians, to appear in Compositio Math.

[2] _ Quantum cohomology of partial flag varieties, in preparation, 2001.

[3] I. Ciocan-Fontanine, Quantum cohomology of flag varieties, Internat. Math. Res. Notices 1995, 263-277. MR 96h:14071

[4] - On quantum cohomology rings of partial flag varieties, Duke Math. J. 98 (1999), 485-524. MR 2000d:14058

[5] S. Fomin, Lecture notes on quantum cohomology of the flag manifold, Publ. Inst. Math. (Beograd) (N.S.) 66(80) (1999), 91-100, Geometric combinatorics (Kotor, 1998). MR 2001g:05106

[6] S. Fomin, S. Gelfand, and A. Postnikov, Quantum Schubert polynomials, J. Amer. Math. Soc. 10 (1997), 565-596. MR 98d:14063

[7] S. Fomin and A. N. Kirillov, Quadratic algebras, Dunkl elements, and Schubert calculus, Advances in geometry, Birkhäuser Boston, Boston, MA, 1999, pp. 147-182. MR 2001a:05152

[8] W. Fulton, Young tableaux, Cambridge University Press, 1997. MR 99f:05119

[9] W. Fulton and R. Pandharipande, Notes on stable maps and quantum cohomology, Algebraic geometry-Santa Cruz 1995, Amer. Math. Soc., Providence, RI, 1997, pp. 45-96. MR 98m:14025

[10] A. Givental and B. Kim, Quantum cohomology of flag manifolds and Toda lattices, Comm. Math. Phys. 168 (1995), 609-641. MR 96c:58027]

[11] B. Kim, Quantum cohomology of flag manifolds $G / B$ and quantum Toda lattices, Ann. of Math. (2) 149 (1999), 129-148. MR 2001c:14081

[12] M. Kontsevich and Yu. Manin, Gromov-Witten classes, quantum cohomology, and enumerative geometry, Mirror symmetry, II, Amer. Math. Soc., Providence, RI, 1997, pp. 607-653.

[13] I. G. Macdonald, Notes on Schubert polynomials, Laboratoire de Combinatoire et d'Informatique Mathématique, Université du Québec à Montréal, 1991.

[14] D. Monk, The geometry of flag manifolds, Proc. London Math. Soc. (3) 9 (1959), 253-286. MR 21:5641

[15] A. Postnikov, On a quantum version of Pieri's formula, Advances in geometry, Birkhäuser Boston, Boston, MA, 1999, pp. 371-383. MR 99m:14096

[16] Y. Ruan and G. Tian, A mathematical theory of quantum cohomology, Math. Res. Lett. 1 (1994), 269-278. MR 95b:58025

Matematisk Institut, Aarhus Universitet, Ny Munkegade, 8000 Århus, C, Denmark

E-mail address: abuch@imf.au.dk 